\title{
Asymmetric Polymerization of Triphenylmethyl Methacrylate Using 9-Alkyl-9-fluorenyllithium-(-)-Sparteine Complexes. Influence of Organolithium Structure on the Propagation Stereochemistry
}

\author{
Tamaki NaKano, Yoshio OKamoto, and Koichi Hatada* \\ Department of Applied Chemistry, School of Engineering, Nagoya University, \\ Furo-cho, Chikusa-ku, Nagoya 464-01, Japan \\ * Department of Synthetic Chemistry, Faculty of Engineering Science, \\ Osaka University, Toyonaka, Osaka 560, Japan
}

(Received January 17, 1995)

\begin{abstract}
Asymmetric anionic polymerization and oligomerization of triphenylmethyl methacrylate (TrMA) were carried out with the complexes of (-)-sparteine (Sp) with 9methyl-9-fluorenyllithium (MeFlLi) and 9-ethyl-9-fluorenyllithium in toluene at $-78^{\circ} \mathrm{C}$ in conjunction with the polymerization of TrMA with $\mathrm{Sp}$-9-fluorenyllithium (FlLi) complex. As well as $\mathrm{Sp}-$ FlLi complex, Sp-MeFlLi complex gave an optically active $\left([\alpha]_{\mathrm{D}}+360^{\circ}\right)$, highly isotactic polymer with one-handed helical conformation; the polymer had a higher molecular weight than that obtained by $\mathrm{Sp}-\mathrm{FlLi}$ under the same reaction conditions. The oligomers obtained at the feed ratio of monomer to initiator of two, three, and five were converted to oligo(methyl methacrylate) [oligo(MMA)]; the oligo(MMA)s were first separated in terms of degree of polymerization and then into diastereomers. The dimer meso $(m)$ and racemo $(r)$ and the trimer $\mathrm{mm}$ and $\mathrm{mr}$ were resolved into enantiomers to determine the ratio of isomers for each oligomer. On the basis of the change in isomer content of the dimer with the change in the feed ratio of monomer to initiator, it was concluded that the dimer anion having $S$ absolute configuration is more active toward TrMA monomer than that having $R$ configuration in both the systems with MeFILi and EtFlLi in contrast to the reaction with $\mathrm{Sp}-\mathrm{FlLi}$ where the $R$-dimer anion is more active. The stereochemistry in the propagation of a trimer anion to a tetramer anion and thereafter appeared similar to the reaction system with $\mathrm{Sp}-\mathrm{FlLi}$ where --- $R R R$--- isomers predominantly propagate. The predominant propagation of the $S$-dimer anion results in a larger amount of the isomeric anions which do not propagate to the optically active polymer.
\end{abstract}

KEY WORDS Triphenylmethyl Methacrylate / Asymmetric Anionic Polymerization / Optically Active Polymer / Helix / (-)-Sparteine / 9-Fluorenyllithium / 9-Methyl-9-fluorenyllithium / 9-Ethyl-9-fluorenyllithium / Oligomer /

One-handed helical, optically active poly(triphenylmethyl methacrylate) [poly(TrMA)] is obtainable by asymmetric anionic polymerization of TrMA. ${ }^{1}$ This polymer is known to have a high chiral recognition ability toward many classes of racemic compounds of chemical and pharmaceutical importance. ${ }^{2}$ Because of this practical application in addition to the interest in the unique polymer structure, various helical polymers have been designed after poly(TrMA). ${ }^{3-6}$ Recently, we reported the oligomerization of TrMA with the complexes of 9-fluorenyllithium (FILi) with (-)-sparteine (Sp), (+)-2,3-dimethoxy-1,4-bis(dimethylamino)butane (DDB), and (+)-1-(2-pyrrolidinylmethyl)pyrrolidine (PMP) and showed the detailed mechanism of the asymmetric polymerization of TrMA. ${ }^{7,8}$ The propagation stereochemistry is largely influenced by the ligand structure and the termination stereochemistry reflects the structure of terminating reagents. ${ }^{9}$ 
In the present study, the influence of organolithium compounds on the polymerization mechanism was investigated; 9-methyl-9fluorenyllithium (MeFlLi) and 9-ethyl-9-fluorenyllithium (EtFlLi) were employed as the derivatives of FlLi. Anionic polymerization and oligomerization of TrMA were carried out with $\mathrm{Sp}-\mathrm{MeFlLi}$ and $\mathrm{Sp}-\mathrm{EtFlLi}$ complexes in toluene at $-78^{\circ} \mathrm{C}$. The oligomers obtained at the feed ratio of monomer to initiator ([TrMA $] /[\mathrm{Li}])$ of 2,3 , and 5 were converted to oligo(methyl methacrylate) [oligo(MMA)]; the oligo(MMA)s were first separated in terms of degree of polymerization (DP) and then into diastereomers. The dimer meso $(m)$ and racemo $(r)$ and the trimer $m m$ and $m r$ obtained at different [TrMA]/[Li] ratios were resolved into enantiomers and the ratio of isomers was determined for each oligomer in order to get information on the propagation stereochemistry at early stages of the polymerization. The difference in the stereochemistry of polymerization between the present systems and the reported systems with FlLi as an organolithium is discussed.

\section{EXPERIMENTAL}

\section{Materials}

Purification of materials other than those described below has been shown in the previous paper. $^{8}$

9-Ethylfluorene (Aldrich) was dried over $\mathrm{CaH}_{2}$ and distilled under a reduced pressure: bp $123-124^{\circ} \mathrm{C}(1 \mathrm{mmHg})$.

9-Methylfluorene was prepared through direct methylation of FlLi with methyl iodide. Fluorene $(10.6 \mathrm{~g}, 64.1 \mathrm{mmol})$ was dissolved in $120 \mathrm{ml}$ of THF under dry nitrogen atmosphere in a 500-ml round-bottomed flask equipped with a three-way stop cock and the solution was cooled at $-78^{\circ} \mathrm{C}$. $n$ - BuLi $(1.37 \mathrm{M}$ in hexane solution, $49.1 \mathrm{ml})$ and then methyl iodide $(5.00 \mathrm{ml}, 80.3 \mathrm{mmol})$ which had been freshly distilled from $\mathrm{CaH}_{2}$ were added to the solution with a syringe with gentle stirring. The solvent was then removed using a rotary evaporator and the residue was dissolved in a mixture of hexane and diethyl ether $(1: 1, \mathrm{v} / \mathrm{v})$. After the solution was washed with water, the solvent was removed to give $11.4 \mathrm{~g}$ of crude product. Analyses of the crude product by ${ }^{1} \mathrm{H}$ NMR spectroscopy and by HPLC using a column $(25 \times 0.46 \mathrm{~cm}$ (i.d.)) packed with cellulose tris(3,5-dimethylphenylcarbamate)-coated macroporous silica gel with a mixture of hexane and 2-propanol $(99: 1, \mathrm{v} / \mathrm{v})$ as eluent (flow rate $\left.0.5 \mathrm{ml} \mathrm{min}^{-1}\right)^{10}$ revealed that three compounds existed: 9-methylfluorene 95\% (elution time $12.2 \mathrm{~min}$ ), 9,9-dimethylfluorene $3 \%(14.4 \mathrm{~min})$, and unreacted fluorene $2 \%$ $(16.7 \mathrm{~min})$. The product was purified by recrystallization first from methanol and then five times from hexane to give $2.10 \mathrm{~g}(18.2 \%)$ of white crystal; purity $100 \%$ (HPLC), mp 46.2- $46.8^{\circ} \mathrm{C}$ (lit. $\left.{ }^{11} 46^{\circ} \mathrm{C}\right) .{ }^{1} \mathrm{H}$ NMR (100 $\mathrm{MHz}, \mathrm{CDCl}_{3}, 60^{\circ} \mathrm{C}$, TMS) $\delta 7.2-8.0(\mathrm{~m}, 8 \mathrm{H}$, aromatic $\mathrm{H}), 3.98(\mathrm{q}, 1 \mathrm{H}, \mathrm{CH}), 1.48(\mathrm{~d}, 3 \mathrm{H}$, $\mathrm{CH}_{3}$ ). Anal. Calcd for $\mathrm{C}_{14} \mathrm{H}_{12}: \mathrm{C}, 93.29 \% ; \mathrm{H}$, $6.71 \%$. Found: C, $93.36 \%$; H, 6.64\%. The low analytical values for carbon pointed out in a literature $^{12}$ for 9-alkylfluorenes was not the case in our study.

As an alternative way to synthesize 9alkylfluorenes, Bordwell and coworkers recommend the reaction of fluorenone with an alkyl Grignard reagent or an alkyllithium followed by hydrogenation of the resulting alcohol. ${ }^{12}$

\section{Measurements}

${ }^{1} \mathrm{H}$ NMR spectra were measured on a JEOL GSX-270 (270 MHz) and a JNM MN-100 $(100 \mathrm{MHz})$ spectrometers. Field desorption (FD) mass spectra were taken using a JEOL DX-HF303 instrument.

HPLC columns and chromatographic conditions are as follows. For GPC separation of oligomers obtained in the system with Sp-MeFlLi, two JASCO Gel-101 columns $(50 \times 0.72$ (i.d.) $\mathrm{cm}$, maximum porosity 3000$)$ connected in series were used with $\mathrm{CHCl}_{3}$ as 
eluent (flow rate $0.5 \mathrm{ml} \mathrm{min}^{-1}$ ). For GPC separation of oligomers obtained in the system with $\mathrm{Sp}-\mathrm{EtFlLi}$, a Gel-101 column was used with $\mathrm{CHCl}_{3}$ as eluent (flow rate $0.5 \mathrm{ml} \mathrm{min}^{-1}$ ). For diastereomeric separation, columns $(25 \times$ 0.46 (i.d.) $\mathrm{cm}, 50 \times 0.72$ (i.d.) $\mathrm{cm}$ ) packed with silica gel were used with a mixture of $n$ chlorobutane $(\mathrm{BuCl})$ and $\mathrm{CH}_{3} \mathrm{CN}$ as an eluent under a gradient condition; from $95 \% \mathrm{BuCl}$ to $50 \% \mathrm{BuCl}$ during a $60-\mathrm{min}$ period (flow rate $0.5 \mathrm{ml} \mathrm{min}^{-1}$ for the former column and $2.4 \mathrm{ml} \mathrm{min}^{-1}$ for the latter one). Resolution of MMA-dimers was done using a chiral column packed with a cellulose tris(3,5-dichlorophenylcarbamate)-coated macroporous silica gel with a mixture of hexane and 2-propanol $(95: 5$, $\mathrm{v} / \mathrm{v}$ ) (flow rate $0.5 \mathrm{ml} \mathrm{min}^{-1}$ ). ${ }^{10}$ Resolution of MMA-trimers was done using a chiral column packed with cellulose tris(3,5-dimethylphenylcarbamate)-coated macroporous silica gel $^{10}$ with the same eluent as for dimers (flow rate $0.5 \mathrm{ml} \mathrm{min}^{-1}$ ).

Molecular mechanics calculation was performed using a CERIUS ${ }^{2}$ program (Molecular Simulations) running on an Indigo ${ }^{2}$-Extreme graphics workstation computer (Silicon Graphics).

Other experimental details have already been described in the previous paper. ${ }^{8}$

\section{RESULTS AND DISCUSSION}

\section{Asymmetric Anionic Polymerization}

The results of polymerization in toluene at $-78^{\circ} \mathrm{C}$ with $\mathrm{Sp}-\mathrm{MeFlLi}$ and $\mathrm{Sp}-$ EtFlLi are summarized in Table I. In both systems with MeFlLi and EtFlLi, the polymer was obtained almost quantitatively. The product in the system with $\mathrm{Sp}-\mathrm{MeFlLi}$ was fractionated with a mixture of benzene and hexane $(1: 1, \mathrm{v} / \mathrm{v})$ $(\mathrm{B} / \mathrm{H})$ to remove oligomers. The polymer obtained with $\mathrm{Sp}$-EtFlLi was insoluble in solvents and was not fractionated with $\mathrm{B} / \mathrm{H}$. The insolubility may be caused by aggregation of the polymer of high degree of polymerization (DP). The both polymers were almost perfectly isotactic as found by ${ }^{1} \mathrm{H}$ NMR analysis of poly(MMA)s derived from the original polymers and the $\mathrm{B} / \mathrm{H}$-insoluble part of the one obtained with $\mathrm{Sp}-\mathrm{MeFlLi}$ showed a high dextrorotation which is comparable to the optical activity of the one-handed helical polymer obtained with $\mathrm{Sp}-\mathrm{FlLi}{ }^{8}$ Although the specific rotation of the polymer obtained in the system with $\mathrm{Sp}-\mathrm{EtFILi}$ could not be precisely determined because of the existence of the insoluble part in a mixture of THF and $\mathrm{CH}_{2} \mathrm{Br}_{2}$, the soluble part showed positive rotation. These results indicate that the complexes with MeFlLi and EtFlLi, as well as $\mathrm{Sp}-\mathrm{FlLi}$, afforded a one-handed helical polymer. However, the DPs of the polymers obtained with Sp-MeFlLi and-EtFlLi were larger and the content of $\mathrm{B} / \mathrm{H}$-soluble oligomer in the product obtained with $\mathrm{Sp}-\mathrm{MeFlLi}$ $(43 \mathrm{wt} \%)$ was higher as compared with the system with Sp-FlLi (18 wt\%), suggesting that the substituent group at 9-position of fluorene

Table I. Asymmetric anionic polymerization of TrMA with Sp-MeFlLi and $\mathrm{Sp}$-EtFlLi complexes in toluene at $-78^{\circ} \mathrm{C}$ for $24 \mathrm{~h}^{\mathrm{a}}$

\begin{tabular}{lllll}
\hline Initiator & $\mathrm{DP}^{\mathrm{b}}$ & $M_{w} / M_{n}{ }^{\mathrm{b}}$ & Tacticity, $\mathrm{mm}^{\mathrm{c}} / \%$ & {$[\alpha]_{\mathrm{D}}^{25 \mathrm{~d}}$} \\
\hline Sp-MeFlLi & $112^{\mathrm{e}}$ & $1.67^{\mathrm{e}}$ & $>99$ & $+360^{\circ}$ \\
Sp-EtFlLi & 118 & 1.82 & $>99$ & Not determined \\
\hline
\end{tabular}

${ }^{a}$ TrMA $1.0 \mathrm{~g}(3.05 \mathrm{mmol})$, toluene $20 \mathrm{ml},[\operatorname{TrMA}] /[\mathrm{Li}]=20$. The yield was quantitative in the both runs. ${ }^{b}$ Determined by GPC of poly(MMA)s derived from poly(TrMA)s. ${ }^{c}$ Determined by ${ }^{1} \mathrm{H}$ NMR of poly(MMA)s derived from poly(TrMA)s. ${ }^{d}$ In a mixture of THF and $\mathrm{CH}_{2} \mathrm{Br}_{2}(1: 5, \mathrm{v} / \mathrm{v}), c 0.5$. ${ }^{\mathrm{e}}$ The original poly(TrMA) sample washed with a mixture of benzene and hexane $(1: 1)$ to remove oligomers: $57 \mathrm{wt} \%$ of the product was benzene-hexane insoluble polymer. 
affected the stereochemistry of propagation in the early stages of the polymerization and larger amount of the species of low activity was produced in the present systems as compared with the system with Sp-FlLi.

\section{Oligomerization and Oligomer Distribution}

Oligomerization reactions were carried out at $[\mathrm{TrMA}] /[\mathrm{Li}]$ ratios of 2,3 , and 5 to clarify the propagation stereochemistry in the early stages of the polymerization. In all cases of the oligomerization, IR spectra of the reaction mixture showed no clear sign of the existence of unreacted monomer, indicating that the reaction was quantitative. Figures 1 and 2 show the GPC curves of oligo(MMA)s derived from

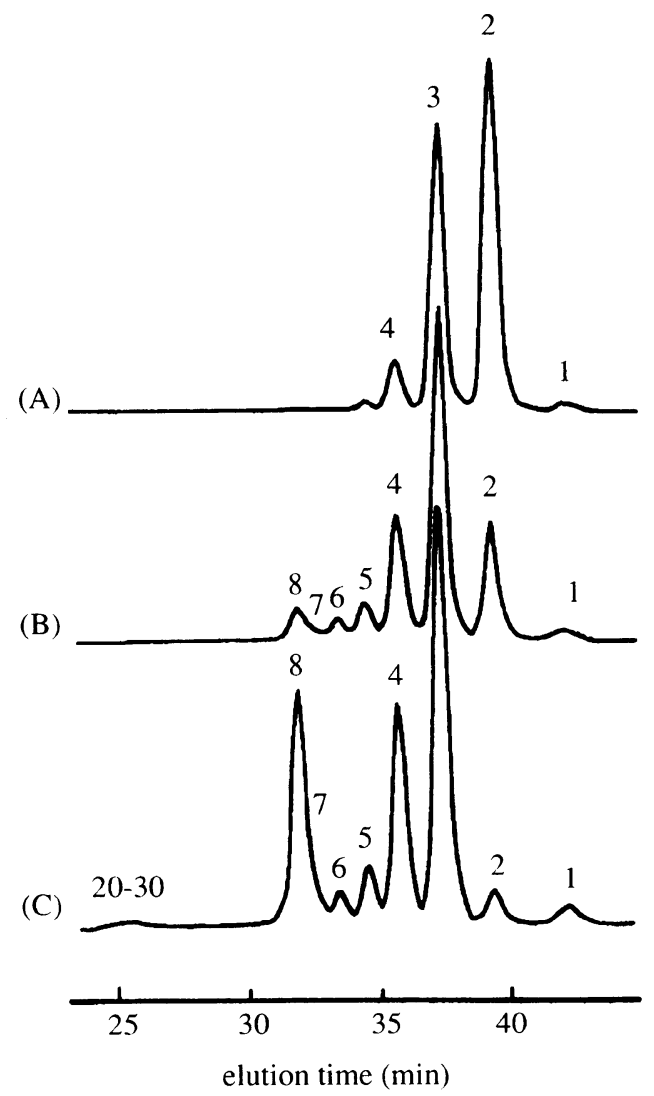

Figure 1. GPC curves of oligo(MMA)s derived from oligo(TrMA)s obtained with $\mathrm{Sp}-\mathrm{MeFlLi}$ complex at [TrMA $] /[\mathrm{Li}]$ ratios of $2(\mathrm{~A}), 3(\mathrm{~B})$, and $5(\mathrm{C})$. The numbers on the curves show the DPs of oligomers.
oligo(TrMA)s obtained with $\mathrm{Sp}-\mathrm{FlLi}$ and $\mathrm{Sp}-\mathrm{EtFlLi}$, respectively. The GPC curves of the original oligo(TrMA)s had similar patterns to those of the corresponding oligo(MMA)s (not shown). The assignment of the peaks was done by comparing the GPC curves with the FD mass spectra of the oligo(MMA)s. The mass spectrum of the oligo(MMA) derived from the oligo(TrMA) obtained with Sp-MeFlLi at [TrMA] $/[\mathrm{Li}]$ ratio of 5 showed peaks at $\mathrm{m} / \mathrm{z}$ of $280,380,480,580,680,780,881,981$, and 1081 and that of the oligo(MMA)s obtained from the system with $\mathrm{Sp}$-EtFlLi showed peaks

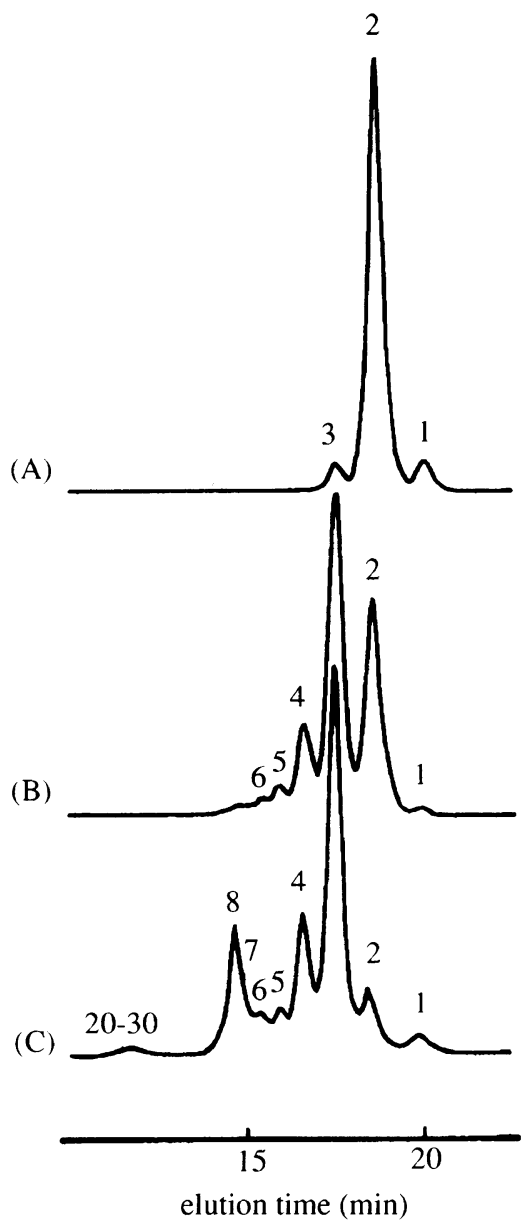

Figure 2. GPC curves of oligo(MMA)s derived from oligo(TrMA)s obtained with $\mathrm{Sp}$-EtFlLi complex at [TrMA]/[Li] ratios of 2 (A), $3(\mathrm{~B})$, and 5 (C). The numbers on the curves show the DPs of oligomers. 
at $m / z$ of $294,394,595,695,795,895,995$, and 1095 corresponding to the molecular weight of the oligomers with DPs of 1 to 9. The intensity ratios of the mass peaks were similar to those of the GPC peaks. In the both spectra, no peaks were seen at $\mathrm{m} / \mathrm{z}$ values of $350,450,550$,----, while those peaks were observed as minor components in the spectra of oligo(MMA)s derived from oligo(TrMA)s prepared with Sp-FlLi. ${ }^{8}$ This point will be discussed later.

In the previous systems with $\mathrm{Sp}-\mathrm{FlLi}$, the existence of unimer was not confirmed, indicating that the unimer anion was much more active than the initiator complex and the dimer anion toward TrMA monomer. ${ }^{8}$ In contrast to this, unimer peaks are clearly seen in the GPC curves. Activity of the unimer anion having 9-substituted fluorenyl group at the $\alpha$-end appears to be lower than that of the unimer anion having a fluorenyl group. Although the oligomer distributions at [TrMA]/ [Li] ratios of 3 and 5 obtained with $\mathrm{Sp}-\mathrm{MeFlLi}$ were similar to those at the same $[\operatorname{TrMA}] /[\mathrm{Li}]$ ratios with $\mathrm{Sp}-\mathrm{EtFlLi}$, the distribution at [TrMA]/[Li] ratio of 2 showed clear discrepancy. In the system with Sp-MeFlLi, the intensity of trimer and higher oligomer peaks was much higher than that in the system with Sp-EtFlLi, suggesting that activity of the dimer anion in the system with $\mathrm{Sp}-\mathrm{MeFlLi}$ is higher than that in the system with Sp-EtFlLi. The distribution at [TrMA]/[Li] ratio of 3 in both the systems was different from the reported distribution in the system with $\mathrm{Sp}$-FlLi: relative amount of tetramer was higher and the existence of oligomers with DP of 6 to 8 was clearer in the present study. Additionally, also at $[\mathrm{TrMA}] /[\mathrm{Li}]$ ratio of 5 , content of tetramer was higher in the present study. As the most characteristic feature of the oligomerization system with $\mathrm{Sp}-\mathrm{FlLi}$ at $[\mathrm{TrMA}] /[\mathrm{Li}]$ ratio of 5 , it has been shown that a small amount of polymer with DP of $20 \sim 30$ is produced in addition to the oligomers with DP of 8 or smaller with the absence of the intermediate oligomers. ${ }^{7,8}$ This feature can also be seen in
Figures 1 and 2. The polymer showed a high dextrorotation in the GPC analysis of original mixture of the oligo(TrMA)s by polarimetric detection (not shown). These results indicate that the reactivity of each oligomer anion depends on its DP and a stable helix starts at DP of $c a .9$ in the systems with $\mathrm{Sp}-\mathrm{MeFlLi}$ and Sp-EtFlLi as well as in the system with Sp-FlLi.

\section{Separation of Diastereomers}

The diastereomers of oligo(MMA)s were separated by HPLC using a silica gel column (Figure 3). The DP of the diastereomer corresponding to each peak in the chromatograms was determined by FD mass spectroscopy. In both the systems with MeFILi and EtFlLi, dimer consisted of two fractions, trimer two predominant fractions, tetramer two major fractions and two minor fractions, and the oligomers with DP of 5 or larger two major fractions for each. It is notable that the

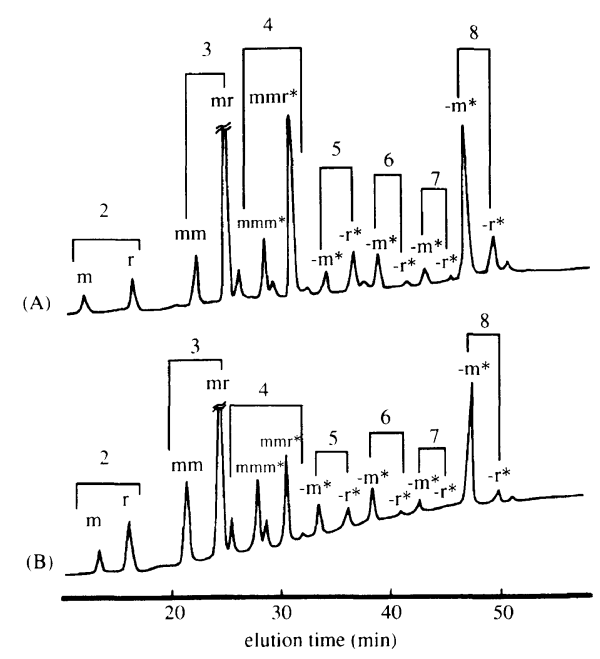

Figure 3. Chromatograms of diastereomeric separation of oligo(MMA)s derived from oligo(TrMA)s obtained with $\mathrm{Sp}-\mathrm{MeFlLi}(\mathrm{A})$ and $\mathrm{Sp}-\mathrm{EtFlLi}(\mathrm{B})$ at [TrMA $] /[\mathrm{Li}]$ ratio of 5. $-m$ and $-r$ mean isotactic oligomers with $m$ and $r$ $\omega$-end diad configuration, respectively. The assignment of asterisked stereosequences was done on the basis of the similarity between the chromatograms in the figure and those reported previously. The stereostructures of the others were assigned by NMR analysis. 

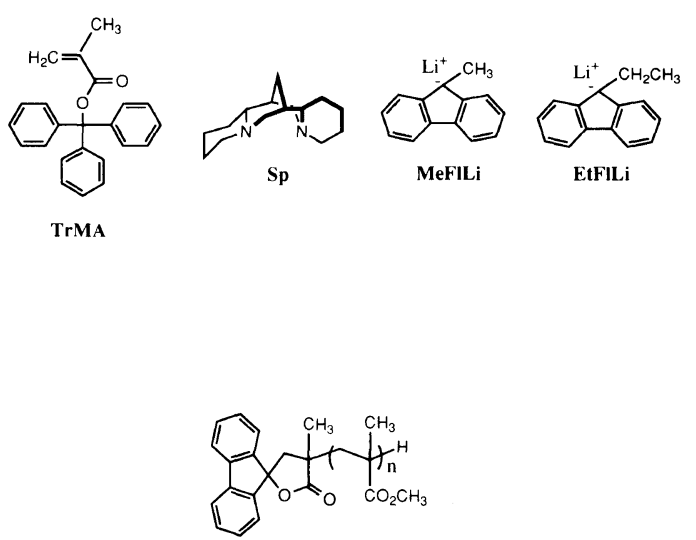

1

chromatograms do not show the peaks of the oligomers of unknown structure which were seen in the analysis of the oligo(MMA)s derived from the oligo(TrMA)s prepared with $\mathrm{Sp}-\mathrm{FlLi}^{8}$ On the basis of the complete absence of the unknown structure in the HPLC analysis in addition to the absence of the molecular ion peaks at $350,450,550,----$ in the mass analysis, we assume structure 1 for the unknown fraction found in the oligomerization system with $\mathrm{Sp}-\mathrm{FlLi}$. It is almost impossible to derive structure 1 from the oligomers having a 9substituted fluorenyl group at the $\alpha$-end.

The stereostructures of the MMA-dimers and -trimers obtained with $\mathrm{Sp}$-FlLi and those of the dimers obtained with Sp-EtFlLi were confirmed by ${ }^{1} \mathrm{H}$ NMR analysis. Though the structure of the trimer obtained with $\mathrm{Sp}-\mathrm{EtFlLi}$ was not determined by NMR, the two main fractions can be assigned to $\mathrm{mm}$ and $\mathrm{mr}$ isomers as the chromatographic pattern of trimer separation in Figure 3 (B) is very similar to that in (A). The assignment of the other oligomers were done on the basis of analogy between the chromatograms in Figure 3 and those of oligo(MMA)s obtained from the reaction with $\mathrm{Sp}-\mathrm{FlLi}$ in which the oligomers with DP of 5 or larger consisted mainly of isotactic isomers. ${ }^{8}$ Hence, the two main fractions of the oligomers with DP of 5 or larger were assigned to the isotactic ones with $m$ and $r$-end diad configurations. This assignment is also consistent with the formation of almost perfectly isotactic polymers in the present study. The two major components of the tetramer were also provisionally assigned to the isotactic ones similarly to the above assignments; this assignment can well explains the stereochemistry of protonation as shown later.

As for the trimer, $r r$ and $r m$ isomers were not obviously found in the both systems with MeFlLi and EtFlLi at all the $[$ TrMA $] /[\mathrm{Li}]$ ratios, suggesting that the formation of $m$ sequence at the $\alpha$-end is highly favored in the propagation of dimer anion to tetramer anion. Though the tetramer in the system with $\mathrm{Sp}-\mathrm{FlLi}$ consisted of many unassigned diastereomers, ${ }^{8}$ in the present study, the tetramers contained two major components which can be isotactic similarly to the other oligomers with DP of 5 and larger. This suggests that the propagation stereochemistry in the step of trimer anion to tetramer anion in the present systems is quite different from that in the system with $\mathrm{Sp}-\mathrm{FlLi}$. Wulff and coworkers reported that in the oligomerization of TrMA with Sp-1,1-diphenylhexyllithium (DPHLi) tetramer consisted of 48 to $53 \%$ of $\mathrm{mmm}$ isomer and 47 to $52 \%$ of $m m r$ isomer. ${ }^{14}$ The propagation stereochemistry in the step of trimer anion to tetramer anion in the system with Sp-DPHLi seems to be similar to that in the systems with $\mathrm{Sp}-\mathrm{MeFlLi}$ and $\mathrm{Sp}-$ EtFlLi. The tertiary alkyllithium compounds (MeFlLi, EtFlLi, DPHLi) probably have a similar steric effect on the propagation stereochemistry of the oligomer anions with relatively low DPs. This could be explained as follows. Based on a computational calculation which suggested that an isotactic poly(TrMA) has a 3.6/1 helical structure ${ }^{15}$ it is assumed that the trimer anion may possibly have a helical conformation. A bulky $\alpha$-end group may enhance the helix formation of the trimer anion and this may be a reason for the higher selectivity on the propagation from the trimer anion to the 
Table II. The ratios of diastereomers of dimer to octamer in the oligomers obtained with $\mathrm{Sp}-\mathrm{MeFlLi}$ and $\mathrm{Sp}-\mathrm{EtFlLi}^{\mathrm{a}}$

\begin{tabular}{|c|c|c|c|c|c|}
\hline \multirow{2}{*}{ Initiator } & [TrMA] & Dimer & Trimer $^{\mathbf{b}}$ & Tetramer & Pentamer \\
\hline & {$[\mathrm{Li}]$} & $m / r$ & $m m / m r$ & $\mathrm{mmm} / \mathrm{mmr}$ & $m m m m / m m m r$ \\
\hline \multirow[t]{3}{*}{ Sp-MeFlLi } & 2 & $17 / 83$ & $18 / 82$ & $61 / 39$ & $\underline{c}^{c}$ \\
\hline & 3 & $28 / 72$ & $15 / 85$ & $42 / 58$ & $61 / 39$ \\
\hline & 5 & $31 / 69$ & $10 / 90$ & $20 / 80$ & $36 / 65$ \\
\hline \multirow[t]{3}{*}{ Sp-EtFlLi } & 2 & $10 / 90$ & $21 / 79$ & ]$^{c}$ & $-^{c}$ \\
\hline & 3 & $22 / 78$ & $15 / 85$ & $49 / 51$ & $61 / 39$ \\
\hline & 5 & $38 / 62$ & $17 / 83$ & $46 / 54$ & $54 / 46$ \\
\hline \multirow{2}{*}{ Initiator } & [TrMA] & Hexamer & \multicolumn{2}{|c|}{ Heptamer } & Octamer \\
\hline & {$[\mathrm{Li}]$} & $\mathrm{mmmmm} / \mathrm{mmmmr}$ & $m m m r$ & $m m m m r$ & $\mathrm{mmmmmmm/mmmmmmr}$ \\
\hline \multirow[t]{3}{*}{$\mathrm{Sp}-\mathrm{MeFlLi}$} & 2 & $-^{c}$ & \multicolumn{2}{|c|}{$\underline{-c}^{c}$} & $-^{\mathrm{c}}$ \\
\hline & 3 & $78 / 22$ & \multicolumn{2}{|c|}{$68 / 32$} & $79 / 21$ \\
\hline & 5 & $86 / 14$ & \multicolumn{2}{|c|}{$78 / 22$} & $86 / 14$ \\
\hline \multirow[t]{3}{*}{ Sp-EtFlLi } & 2 & $-^{c}$ & \multicolumn{2}{|c|}{-c } & ${ }^{c}$ \\
\hline & 3 & $73 / 27$ & \multicolumn{2}{|c|}{$58 / 42$} & $70 / 30$ \\
\hline & 5 & $74 / 26$ & \multicolumn{2}{|c|}{$64 / 36$} & $87 / 13$ \\
\hline
\end{tabular}

${ }^{a}$ Determined by HPLC analysis of oligo(MMA)s derived from oligo(TrMA)s with a UV (254 nm) detector. ${ }^{b}$ The $r r$ and $r m$ isomers were not obviously found in the HPLC separation and ${ }^{1} \mathrm{H}$ NMR analysis. ${ }^{\mathrm{c}}$ The abundance of these oligomers was too low to know the ratios of isomers.

tetramer anion in the present and the DPHLi systems than that in the FlLi system.

\section{Stereochemistry of Protonation of Oligomer Anions}

The ratios of the diastereomers in each oligomer are summarized in Table II. For most isomers of dimer, trimer, pentamer, and higher oligomers at all the $[\mathrm{TrMA}] /[\mathrm{Li}]$ ratios in both the systems, the ratio of isomers having $m$ $\omega$-end diad configuration to that having $r$ $\omega$-end configuration was similar to the corresponding ratio in the system with $\mathrm{Sp}$ $\mathrm{FlLi}^{8}{ }^{8}$ stereochemistry of protonation (termination) seems not to be greatly affected by the substituents at the $\alpha$-end of oligomer anions. Exceptionally, the pentamer in the system with $\mathrm{Sp}-\mathrm{MeFlLi}$ at [TrMA]/[Li] ratio of 5 had $65 \%$ of $m m r$ isomer; this result is contrast to the predominant $m$-protonation for the pentamer anion in the system with Sp-FlLi.
It has been revealed for the reaction system with $\mathrm{Sp}-\mathrm{FlLi}$ that the protonation stereochemistry of pentamer and higher oligomer anions depends on the absolute configuration of the main chain; i.e., $m$-protonation predominates for the isomeric anion of --RRR-configuration and $r$-protonation for those of --SSS-- configuration leading $R \omega$-end absolute configuration for the both isomeric anions. ${ }^{8}$ Probably, the pentamer anion in the system with $\mathrm{Sp}-\mathrm{MeFlLi}$ at $[\mathrm{TrMA}] /[\mathrm{Li}]$ ratio of 5 contains a higher amount of $S S S S$ - $^{-}$anion than that in $\mathrm{Sp}-$ FlLi system.

Assuming the dependence of the protonation stereochemistry on the main chain configuration is applicable also for the tetramer anion, one can estimate that the tetramer anion in the $\mathrm{Sp}-\mathrm{MeFlLi}$ system contains a significant amount of $S S S$ - $^{-}$anion and the amount increases with the increase in $[\operatorname{TrMA}] /[\mathrm{Li}]$ ratio on the basis of the observation that $\mathrm{mmr}$ 
Table III. ${ }^{1} \mathrm{H}$ NMR chemical shifts $(\delta$, ppm) of the diastereomeric MMA-dimers and trimers having a 9-methyl- or 9-ethylfluorenyl group ${ }^{a}$

\begin{tabular}{|c|c|c|c|c|c|c|c|c|c|c|}
\hline & \multicolumn{2}{|c|}{ Fluorenyl } & \multicolumn{2}{|c|}{$\alpha_{1}$} & \multicolumn{2}{|c|}{$\alpha_{2}$} & \multicolumn{2}{|c|}{$\omega_{1}$} & \multirow{2}{*}{$\begin{array}{l}\omega \text {-End } \\
\text { methine }^{\mathrm{f}}\end{array}$} & \multirow{2}{*}{$\mathrm{OCH}_{3}{ }^{\mathrm{g}}$} \\
\hline & $\mathrm{CH}_{2}$ & $\mathrm{CH}_{3}$ & $\mathrm{CH}_{2}{ }^{\mathrm{b}}$ & $\alpha-\mathrm{CH}_{3}{ }^{\mathrm{c}}$ & $\mathrm{CH}_{2}{ }^{\mathrm{b}}$ & $\alpha-\mathrm{CH}_{3}{ }^{\mathrm{c}}$ & $\mathrm{CH}_{2}{ }^{\mathrm{d}}$ & $\alpha-\mathrm{CH}_{3}{ }^{\mathrm{e}}$ & & \\
\hline \multicolumn{11}{|c|}{$\alpha$-End group: 9-methylfluorenyl } \\
\hline \multirow[t]{2}{*}{ dimer $m$} & & $1.39^{\mathrm{h}}$ & 2.34 & 0.29 & & & 1.47 & 0.94 & 2.09 & $3.56,3.14$ \\
\hline & & & 2.76 & & & & 1.73 & & & \\
\hline \multirow[t]{2}{*}{$r$} & & $1.39^{\mathrm{h}}$ & 2.34 & 0.34 & & & 1.10 & 0.96 & 2.30 & $3.54,3.15$ \\
\hline & & & 2.77 & & & & 2.06 & & & \\
\hline \multicolumn{11}{|c|}{$\alpha$-End group: 9-ethylfluorenyl } \\
\hline \multirow[t]{2}{*}{ dimer $m$} & $1.93^{\mathrm{i}}$ & $0.11^{\mathrm{j}}$ & 2.32 & 0.28 & & & 1.47 & 0.94 & 2.11 & $3.56,3.14$ \\
\hline & & & 2.74 & & & & 1.74 & & & \\
\hline \multirow[t]{2}{*}{$r$} & $1.93^{\mathrm{i}}$ & $0.10^{\mathrm{j}}$ & 2.32 & 0.34 & & & 1.11 & 0.95 & 2.31 & $3.54,3.15$ \\
\hline & & & 2.76 & & & & 2.07 & & & \\
\hline \multicolumn{11}{|c|}{$\alpha$-End group: 9-methylfluorenyl } \\
\hline \multirow[t]{2}{*}{ trimer $\mathrm{mm}$} & & $1.37^{\mathrm{h}}$ & 2.32 & 0.27 & 2.04 & 0.94 & 1.47 & 0.94 & 2.34 & $3.60,3.51,3.15$ \\
\hline & & & 2.74 & & 1.55 & & 1.74 & & & \\
\hline \multirow[t]{2}{*}{$m r$} & & $1.36^{\mathrm{h}}$ & 2.33 & 0.27 & 1.58 & 1.03 & 1.11 & 0.95 & 2.37 & $3.58,3.47,3.11$ \\
\hline & & & 2.75 & & 2.06 & & 2.07 & & & \\
\hline
\end{tabular}

${ }^{\text {a }}$ The spectra were taken in $\mathrm{CDCl}_{3}$ at $35^{\circ} \mathrm{C}$ using a $270 \mathrm{MHz}$ spectrometer. Aromatic protons based on 9-methyl- or 9-ethylfluorenyl group resonated in the range of 7.21 to $7.72 \mathrm{ppm}$ (multiplet). ${ }^{\mathrm{b}} \mathrm{AB}$ quartet. ${ }^{2} J$ coupling constants between the two geminal protons were in the range of 14 to $15 \mathrm{~Hz}$. ${ }^{\mathrm{c}}$ Singlet. ${ }^{\mathrm{d}}$ Double quartet. ${ }^{2} J$ coupling constants between the two geminal protons were $c a .14 \mathrm{~Hz} .{ }^{3} \mathrm{~J}$ coupling constansts between the two methylene proton and the $\omega$-end methine proton were $c a .3 \mathrm{~Hz}$ and $c a .8-9 \mathrm{~Hz}$. ${ }^{\mathrm{e}}$ Doublet. ${ }^{3} \mathrm{~J}$ coupling constants between the methyl protons and $\omega$-end methine proton were $c a .7 \mathrm{~Hz} .{ }^{\mathrm{f}}$ Multiplet. ${ }^{\mathrm{g}}$ Singlet. The signals at around $3.1 \mathrm{ppm}$ appear to be based on $\alpha_{1}-\mathrm{OCH}_{3}$ group (see the text). ${ }^{\mathrm{h}}$ Singlet. ${ }^{\mathrm{i}}$ Qualtet. ${ }^{\mathrm{j}}$ Triplet.

isomer increases with increasing [TrMA]/[Li] ratio.

\section{Structure of MMA-Dimer and-Trimer}

The ${ }^{1} \mathrm{H}$ NMR chemical shifts and coupling constants are summarized in Table III and the numbering system of monomeric units is shown in Scheme 1. The assignments of the signals were done on the basis of the two dimensional spectra taken under the same conditions as those applied to the structural study of the oligo(MMA)s having a $t$-butyl group at the $\alpha$-end. ${ }^{16}$ The difference in chemical shifts of the geminal methylene protons of $\omega_{1}$-monomeric unit was 0.26 to $0.27 \mathrm{ppm}$ for $m$ diad and 0.96 to $1.02 \mathrm{ppm}$ for $r$ diad, that of $\alpha_{2}$-monomeric unit of trimer $0.49 \mathrm{ppm}$ for $m$, and that of $\alpha_{1}$-monomeric unit 0.42 to $0.44 \mathrm{ppm}$ almost independently on the stereostructure. These values are close to those of the oligomers having
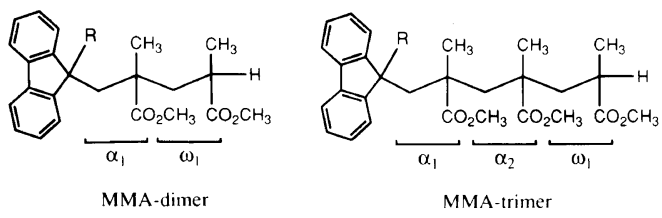

Scheme 1. Structures of MMA-dimer and -trimer having a 9-substituted fluorenyl group at the $\alpha$-end with the numbering system for monomeric units.

a fluorenyl group at the $\alpha$-end. ${ }^{8}$

As the examples of spectra, those of MMA-dimers ${ }^{17}$ and a trimer are shown in Figure 4. Several remarkable differences can be seen between the spectra of the oligo(MMA)s having a 9-substituted fluorenyl group and the dimer having a fluorenyl group as follows. The $\alpha_{1}-\mathrm{CH}_{3}$ protons of the dimers and the trimer with 9-substituted fluorenyl group resonated in the range of 0.27 to $0.34 \mathrm{ppm}$ which was higher by $c a .1 \mathrm{ppm}$ than the chemical shift range for 


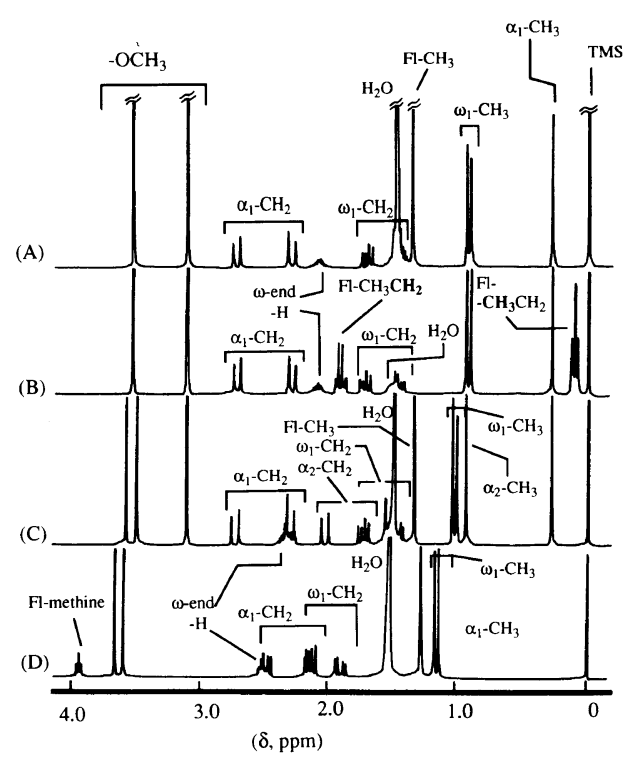

Figure 4. ${ }^{1} \mathrm{H}$ NMR spectra of MMA-dimer $m$ having a 9-methylfluorenyl group at the $\alpha$-end (A), MMA-dimer $m$ having a 9-ethylfluorenyl group (B), MMA-trimer $\mathrm{mm}$ having a 9-methylfluorenyl group (C), and MMA-dimer $m$ having a fluorenyl group ${ }^{17}$ (D). $\left[270 \mathrm{MHz}, \mathrm{CDCl}_{3}, 35^{\circ} \mathrm{C}\right.$, TMS]

the $\alpha$-methyl protons of the dimer having a fluorenyl group. The methyl protons of the 9-ethyl group of the dimer resonated in an unusually high field. The $\alpha_{1}-\mathrm{CH}_{2}$ protons of the oligomer with a 9-substituted fluorenyl group resonated in a lower field than those of the dimer with a fluorenyl group. The signal of one of the methoxy groups whose assignments to the monomeric units could not be done by COSY spectra shifted to higher field by $c a .0 .5 \mathrm{ppm}$ with the replacement of a hydrogen at the 9-position by a methyl or an ethyl group. These observations suggest that some conformational differences exist between the MMA-dimer and -trimer having a 9substituted fluorenyl group and those having a non-substituted fluorenyl group as a result of steric effect of the 9-substituent group. Furthermore, on this basis, it can be assumed that the TrMA-dimer and -trimer anions in the reaction system with $\mathrm{Sp}-\mathrm{MeFlLi}$ and $\mathrm{Sp}-\mathrm{EtFl-}$ Li may have some different conformations
(A)
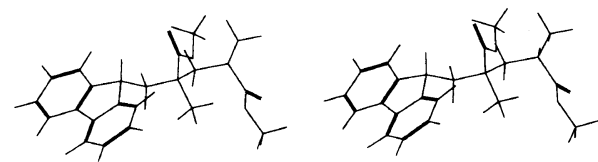

(B)
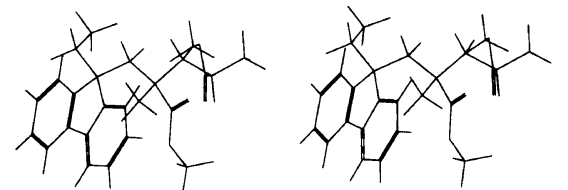

Figure 5. Stereoviews of energy-minimized structures of MMA-dimer having a 9-fluorenyl group at the $\alpha$-end (A) and that having a 9-ethylfluorenyl group (B).

from their counterparts in the reaction system with Sp-FlLi.

Molecular mechanics calculation on the MMA-dimers $m$ having a 9-fluorenyl and 9-ethylfluorenyl groups suggested conformational differences between the two dimers (Figure 5). The conformation of the dimer having a 9-methylfluorenyl group well explains the above unusual chemical shifts. The model shows that the $\alpha$-methyl and methoxy groups of $\alpha_{1}$-monomeric unit and the methyl moiety of 9-ethyl group are likely to experience the shielding effect of the aromatic ring while the $\alpha_{1}$-methylene protons appear to be deshielded. The methoxy methyl signal at ca.3.1 to $3.15 \mathrm{ppm}$ can be assigned to that of the $\alpha_{1}$-methoxy group as based on the model.

\section{Resolution of MMA-Dimer and-Trimer}

The diastereomers of MMA-dimers and -trimers were resolved using a chiral HPLC column and the ratio of enantiomers in each diastereomer was determined in order to get detailed information on the propagation stereochemistry. Figures 6 and 7 show the chromatograms. The dimers in addition to the trimers with a 9-ethylfluorenyl group were perfectly resolved and the trimers with a 9-methylfluorenyl group were only partially resolved. However, even in the latter case, the separation was sufficient enough to estimate enantiomer components. 
(A)

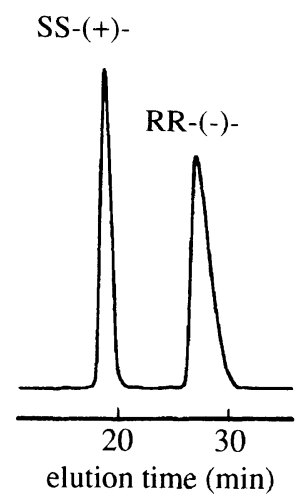

(B)

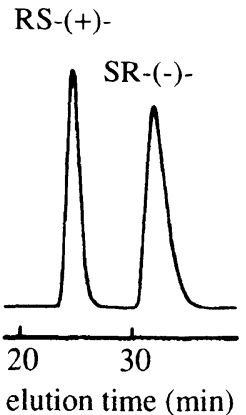

(C)

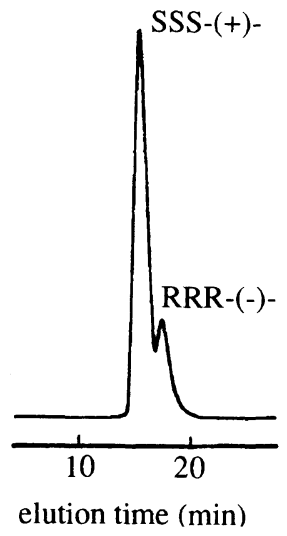

SSR-(-)-

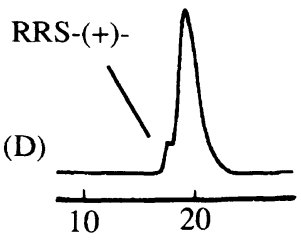

elution time ( $\min )$

Figure 6. HPLC resolution chromatograms of oligo(MMA)s having a 9-methylfluorenyl group at the $\alpha$-end: dimer $m(\mathrm{~A})$, dimer $r(\mathrm{~B})$, trimer $m m(\mathrm{C})$, and trimer $m r(\mathrm{D})$. The oligo(MMA)s were derived from the corresponding oligo(TrMA)s obtained with Sp-MeFlLi at [TrMA]/[Li] ratio of 2.

(A)

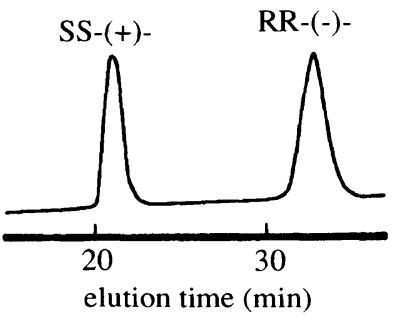

(C)

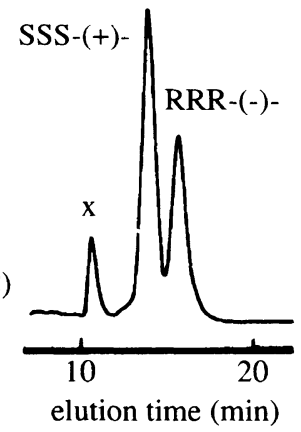

(B)

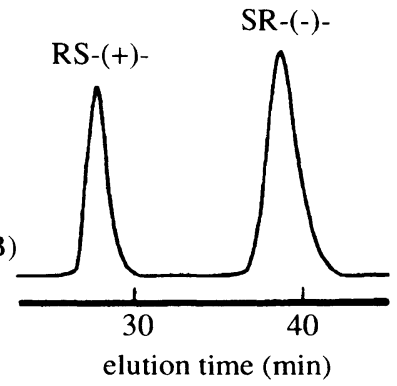

(D)

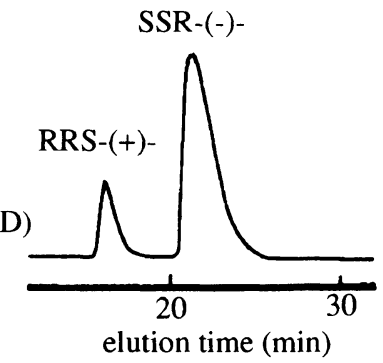

Figure 7. HPLC resolution chromatograms of the oligo(MMA)s having a 9-ethylfluorenyl group at the $\alpha$-end: dimer $m(\mathrm{~A})$, dimer $r(\mathrm{~B})$, trimer $m m(\mathrm{C})$, and trimer $m r(\mathrm{D})$. The oligo(MMA)s were derived from the corresponding oligo(TrMA)s obtained with Sp-EtFlLi at [TrMA]/[Li] ratio of 2.

The assignment of the enantiomers was done on the basis of the relationship between the sign of optical rotation and the absolute configuration of the model compounds illustrated in Chart 1; $(S)$-configuration corresponds to dextrorotation. ${ }^{19}$ This way of assignment based on the assumption that the sign of optical rotation of MMA-dimer and -trimer is determined by the $\omega$-end absolute configuration has been proved to be consistent with the 
more dependable assignment in the previous study for the MMA-dimer having a fluorenyl group at the $\alpha$-end. ${ }^{8}$

The ratio of the isomers are summarized in Table IV. In the both systems, the ratio of the $S$-isomer, which means the sum of $S S$ and $S R$ isomers, decreased with the increasing [TrMA $] /[\mathrm{Li}]$ ratio, indicating that $S{ }^{-}$-dimer anion which produces $S S$ and $S R$ isomers though protonation is more active than $R$ - $^{-}$anion. The stereospecificity in this step of the polymerization is opposite to that in the

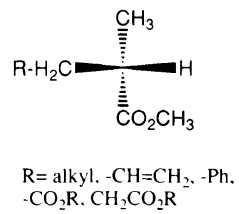

Chart 1. Model compounds of oligo(MMA). system with $\mathrm{Sp}-\mathrm{FlLi}$. This is considered to be correlated with the conformational difference of dimers having a 9-substituted and nonsubstituted fluorenyl group. However, the two types of complexes proposed for the complexes of the oligomer anion with $\mathrm{Sp}$ in the previous study $^{8}$ seem not necessary to explain the present results.

The trimers were rich in the $S S$-isomer, which is the sum of $S S S$ and $S S R$ isomers, in the both systems and the ratio of $R R$-decreased with the increasing [TrMA] $/[\mathrm{Li}]$ ratio leading to the purity of $S S$-isomer of $98 \%$ and $96 \%$ respectively in the systems with MeFlLi and EtFlLi at $[$ TrMA $] /[\mathrm{Li}]$ ratio of 5 . This indicates that the $R R-^{-}$trimer anion which produces $R R R$ and $R R S$ trimers by protonation is more active toward TrMA monomer than the $S S$ - $^{-}$anion which produces $S S S$ and

Table IV. The content of isomers of dimer and trimer obtained with Sp-MeFlLi and Sp-EtFlLi

\begin{tabular}{|c|c|c|c|c|c|c|}
\hline \multirow{3}{*}{ Initiator } & \multirow{3}{*}{$\frac{[\text { TrMA }]}{[\mathrm{Li}]}$} & \multicolumn{5}{|c|}{ Isomers of dimer } \\
\hline & & \multicolumn{2}{|c|}{$m$} & \multicolumn{2}{|c|}{$r$} & \multirow{2}{*}{$S-/ R^{-}$} \\
\hline & & $S S-(+)-$ & $R R-(-)-$ & $R S-(+)-$ & $S R-(-)-$ & \\
\hline \multirow[t]{3}{*}{ Sp-MeFlLi } & 2 & 5 & 10 & 38 & 47 & $52 / 48$ \\
\hline & 3 & 11 & 18 & 43 & 28 & $38 / 62$ \\
\hline & 5 & 13 & 18 & 45 & 24 & $37 / 63$ \\
\hline \multirow[t]{3}{*}{ Sp-EtFlLi } & 2 & 4 & 6 & 33 & 57 & $61 / 39$ \\
\hline & 3 & 9 & 13 & 35 & 43 & $52 / 48$ \\
\hline & 5 & 14 & 24 & 32 & 30 & $44 / 56$ \\
\hline
\end{tabular}

\begin{tabular}{|c|c|c|c|c|c|c|}
\hline \multirow{3}{*}{ Initiator } & \multirow{3}{*}{$\frac{[\operatorname{TrMA}]}{[\mathrm{Li}]}$} & \multicolumn{5}{|c|}{ Isomers of trimer } \\
\hline & & \multicolumn{2}{|c|}{$m m$} & \multicolumn{2}{|c|}{$m r$} & \multirow{2}{*}{$S S-/ R R-^{c}$} \\
\hline & & $S S S-(+)-$ & $R R R-(-)-$ & $R R S-(+)-$ & $S S R-(-)-$ & \\
\hline \multirow[t]{3}{*}{ Sp-MeFlLi } & 2 & 12 & 6 & 9 & 73 & $85 / 15$ \\
\hline & 3 & 12 & 3 & 5 & 80 & $92 / 8$ \\
\hline & 5 & 10 & 1 & 1 & 88 & $98 / 2$ \\
\hline \multirow[t]{3}{*}{ Sp-EtFlLi } & 2 & 12 & 9 & 14 & 65 & $77 / 23$ \\
\hline & 3 & 12 & 3 & 6 & 79 & $91 / 9$ \\
\hline & 5 & 16 & 1 & 3 & 80 & $96 / 4$ \\
\hline
\end{tabular}

${ }^{a}$ Determined by HPLC resolution of individual diastereomers with a UV detector $(254 \mathrm{~nm})$. ${ }^{b}$ The ratio of $[(S, S)$-isomer $+(S, R)$-isomer $]$ to $[(R, R)$-isomer $+(R, S)$-isomer $]{ }^{\mathrm{c}}$ The ratio of $[(S, S, S)$-isomer $+(S, S, R)$-isomer $]$ to $[(R, R, R)$-isomer $+(R, R, S)$-isomer $]$. 
$S S R$ trimers by protonation. This result is similar to that observed for the system with $\mathrm{Sp}-\mathrm{FlLi}$. Although a significant amount of the less active $S S$ - $^{-}$anion should propagate to the tetramer and pentamer anions because the trimers are rich in the $S \mathrm{~S}^{-}{ }^{-}$anion, the resulting tetramer and pentamer anions will remain without any more significant propagation. This interpretation is consistent with the assumption (stereochemistry of protonation) that the tetramer and pentamer appear to contain a significant amount of the SSS-- and SSSSisomeric anions, respectively. The stereospecificity in the steps to higher oligomers in the present reaction systems can reasonably be assumed to be similar to that of the reported systems yielding the --- $R R R$--- configuration $^{8}$ because the stereochemistry of the protonation of hexamer to octamer anions was similar to that in the system with Sp-FlLi where $R$-absolute configuration is predominantly formed at the $\omega$-end leading to a predominant $m$-diad at the $\omega$-end.

As pointed out in the first part of this paper, a polymer with a higher molecular weight was produced in the present study compared to the reaction with $\mathrm{Sp}-\mathrm{FlLi}$. This can be interpreted in terms of the results shown above. In the step from the dimer anion to the trimer anion, the $S$ - $^{-}$anion predominantly propagates resulting in the formation of a significant amount of the $S S-{ }^{-}$trimer anion, the $S S S-{ }^{-}$tetramer anion, and the SSSS- - pentamer anion which are considered not to propagate to a high polymer. Consequently, the amount of the propagating species for the optically active polymer is smaller than that in the system with Sp-FlLi and this results in a higher molecular weight of the polymer.

Acknowledgement. This work was supported in part by a Grant-in-Aid for Scientific
Research (No. 04403021) from the Ministry of Education, Science, and Culture of Japan.

\section{REFERENCES AND NOTES}

1. Y. Okamoto, K. Suzuki, K. Ohta, K. Hatada, and H. Yuki, J. Am. Chem. Soc., 101, 4796 (1979); Y. Okamoto. H. Shohi, and H. Yuki, J. Polym. Sci., Polym. Lett. Ed., 21, 601 (1983).

2. Y. Okamoto and K. Hatada, J. Liq. Chromatogr., 9, 369 (1986).

3. Y. Okamoto and T. Nakano, Chem. Rev., 94, 349 (1994).

4. For a crotonate, see K. Ute, T. Asada, Y. Nabeshima, and K. Hatada, Macromolecules, 26, 7086 (1994).

5. For acrylonitrile derivatives, see G. Wulff and Y. Wu, Makromol. Chem., 191, 2993 (1990); ibid, 191, 3005 (1990).

6. For acrylamides, see Y. Okamoto, H. Hayashida, and K. Hatada, Polym. J., 21, 543 (1989).

7. Y. Okamoto, E. Yashima, T. Nakano, and K. Hatada, Chem. Lett., 759 (1987).

8. T. Nakano, Y. Okamoto, and K. Hatada, J. Am. Chem. Soc., 114, 1318 (1992).

9. T. Nakano, Y. Okamoto, and K. Hatada, Polym. J., 27, 882 (1995).

10. Y. Okamoto, M. Kawashima, and K. Hatada, $J$. Chromatogr., 363, 173 (1986); Y. Okamoto and Y. Kaida, J. Chromatogr. A, 666, 403 (1994).

11. F. G. Bordwell, G. E. Drucker, and G. J. McCollum, J. Org. Chem., 47, 2504 (1982).

12. B. J. Tabner and Y. Walker, J. Chem. Soc., Perkin Trans. 2, 4, 445 (1972).

13. Y. Okamoto, H. Mohri, T. Nakano, and K. Hatada, Chirality, 3, 277 (1991).

14. G. Wulff, R. Sczepan, and A. Steigel, Tetrahedron Lett., 27, 1991 (1986)

15. L. Cavallo, P. Corradini, and M. Vacatello, Polym. Commun., 30, 236 (1989)

16. (a) K. Ute, T. Nishimura, Y. Matsuura, and K. Hatada, Polym. J., 21, 231 (1989). (b) K. Ute, T. Nishimura, and K. Hatada, Polym. J., 21, 1027 (1989).

17. The MMA-dimer $m$ sample was available from our previous work. The spectral data of the dimer are available in ref 8 and 18 .

18. T. Nakano, K. Ute, Y. Okamoto, Y. Matsuura, and K. Hatada, Polym. J., 21, 935 (1989).

19. H. B. Kagan and J. Jaques, "Stereochemistry," Vol. 4, Geroge Thieme Publishers, Stuttgart, 1977, pp $79-83$. 